\title{
Effect of strain rate on the yielding mechanism of amorphous metal foam
}

\author{
Joseph P. Schramm, ${ }^{1, a)}$ Marios D. Demetriou, ${ }^{1}$ William L. Johnson, ${ }^{1}$ Benny Poon, ${ }^{2}$ \\ Guruswami Ravichandran, ${ }^{2}$ and Daniel Rittel ${ }^{3}$ \\ ${ }^{1}$ Keck Engineering Laboratories, California Institute of Technology, Pasadena, California 91125, USA \\ ${ }^{2}$ Graduate Aerospace Laboratories, California Institute of Technology, Pasadena, Pasadena, \\ California 91125, USA \\ ${ }^{3}$ Faculty of Mechanical Engineering, Technion, 32000 Haifa, Israel
}

(Received 23 September 2009; accepted 30 November 2009; published online 14 January 2010)

\begin{abstract}
Stochastic amorphous $\mathrm{Pd}_{43} \mathrm{Ni}_{10} \mathrm{Cu}_{27} \mathrm{P}_{20}$ foams were tested in quasistatic and dynamic loading. The strength/porosity relations show distinct slopes for the two loading conditions, suggesting a strain-rate-induced change in the foam yielding mechanism. The strength/porosity correlation of the dynamic test data along with microscopy assessments support that dynamic foam yielding is dominated by plasticity rather than elastic buckling, the mechanism previously identified to control quasistatic yielding. The strain-rate-induced shift in the foam yielding mechanism is attributed to the rate of loading approaching the rate of sound wave propagation across intracellular membranes, thereby suppressing elastic buckling and promoting plastic yielding. () 2010 American Institute of Physics. [doi:10.1063/1.3279132]
\end{abstract}

Recent progress in the processing of metallic glasses has led to the development of open- and closed-cell amorphous metal foams fabricated from various alloys via a variety of methods. ${ }^{1-6}$ Because of the unique mechanical properties of amorphous metals (e.g., high strength and elasticity, broadly varying toughness, and lack of ductility), ${ }^{7}$ amorphous metal foams inherit a set of mechanical properties not previously seen in porous solids of any kind. Specifically, cellular structures consisting of struts thinner than the process zone size of the amorphous metal were found to be heavily deformable, as catastrophic failure due to global brittle fracture is avoided ${ }^{8,9}$ Highly stochastic cellular structures consisting of struts with broadly varying thicknesses and aspect ratios were found to yield by percolation of elastic buckling, a consequence of the high elastic limit of the amorphous metal. ${ }^{10}$ Elastic yielding gives rise to a steep strength/ porosity relation, resulting in very high strengths at high relative densities, ${ }^{11}$ however, as the limit of cooperative buckling is approached at low relative densities, the attainable foam strengths are rather low. Interestingly, by matching the structural scales controlling these two yielding mechanisms (brittle fracture and buckling percolation), i.e., by attaining cellular structures consisting of thin struts with uniform slenderness ratios, foams of low relative densities $(<10 \%)$ yield plastically. ${ }^{12}$ Consequently, such foams are able to inherit the high plastic yield strength of the amorphous metal at very high porosities, and emerge among the strongest foams of any kind. The strain-rate sensitivity of these porous solids has not yet been investigated. Monolithic (pore-free) amorphous metals are known to be strain-rate insensitive. ${ }^{13,14}$ In this letter we demonstrate that unlike the parent solid, the yielding mechanism of a stochastic amorphous-metal cellular structure is sensitive to the rate of the applied strain. Hence, a drastic change in strain rate results in a shift in the overall strength/porosity relation.

Amorphous $\mathrm{Pd}_{43} \mathrm{Ni}_{10} \mathrm{Cu}_{27} \mathrm{P}_{20}$ foam specimens with highly stochastic closed-cell porosity were utilized. The

${ }^{a)}$ Electronic mail: schramm@caltech.edu. foams were produced by thermoplastically expanding entrained bubbles in the supercooled liquid region, as described elsewhere. ${ }^{15}$ The statistical nature of these foams has been analyzed in prior work. ${ }^{16}$ Dynamic and quasistatic compression tests were carried out on plane-parallel cylindrical specimens with relative densities ranging from $13 \%$ to $65 \%$. For the dynamic tests, specimens with diameter of about $12 \mathrm{~mm}$ and height of about $6 \mathrm{~mm}$ were used. A representative dynamic-test specimen is shown in Fig. 1(a). For the quasistatic tests, specimens with aspect ratios between 1.0 and 1.5 were used. Porosities of all samples were measured using the Archimedes method, as given in ASTM standard C693-93.

For quasistatic tests, a screw-driven Instron was used. Displacements were measured using a linear variable differential transformer. Strain rates for quasistatic tests ranged

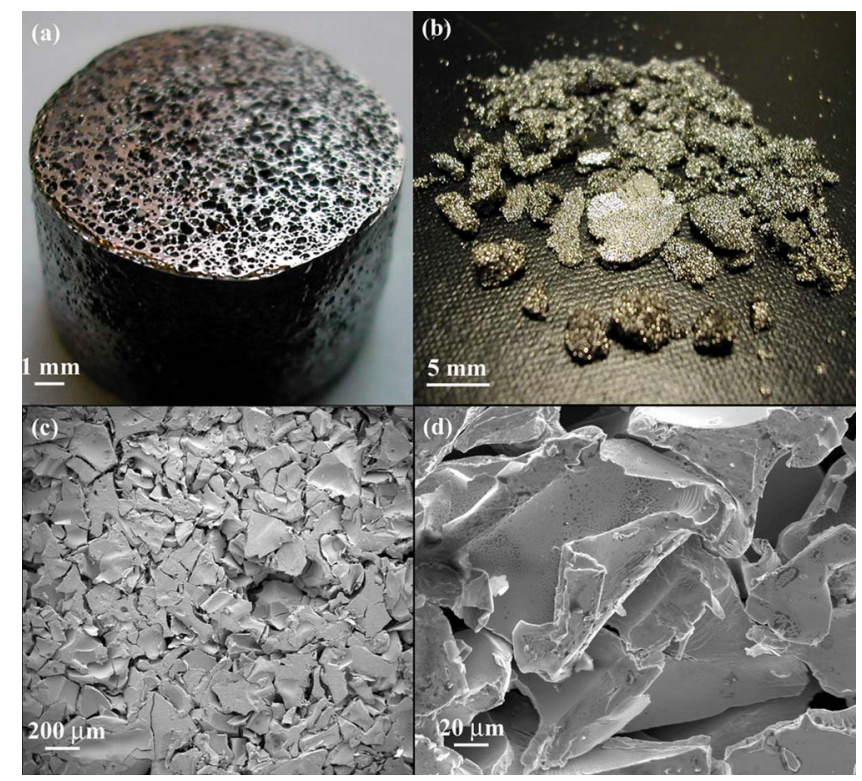

FIG. 1. (Color online) (a) Foam specimen as prepared for dynamic compression. (b) Foam specimen after dynamic compression showing several densified pieces among other crushed pieces. [(c) and (d)] Electron micrographs of a densified piece of a dynamically compressed foam. 


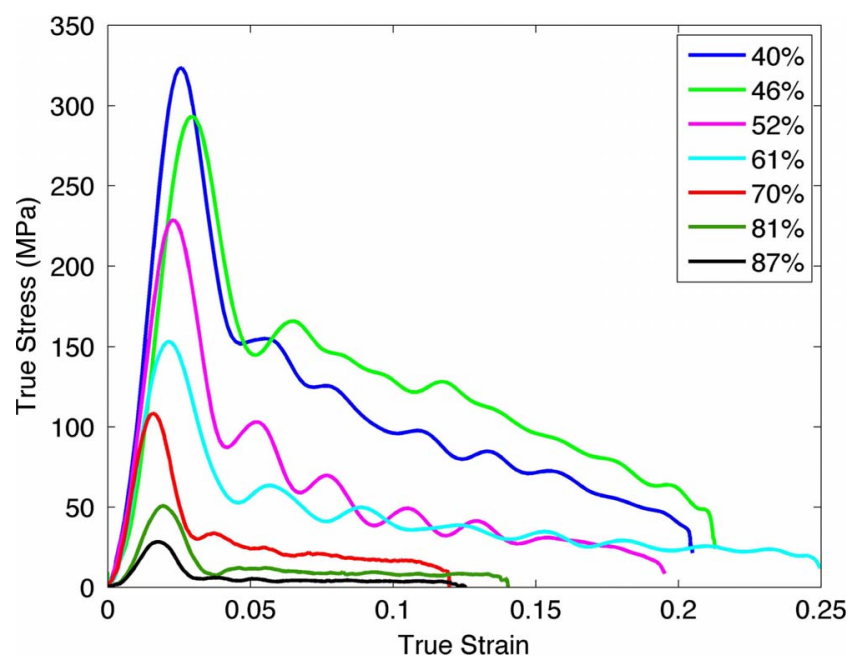

FIG. 2. (Color online) Dynamic stress-strain response of foams of varying relative density (reported in \% porosity) for strain rates of 3000-3500 s $\mathrm{s}^{-1}$.

between $10^{-3}$ and $10^{-4} \mathrm{~s}^{-1}$. Dynamic compression was carried out using a $19.05 \mathrm{~mm}$ diameter split Hopkinson (Kolsky) pressure bar made of C300 Maraging steel. Strain rates ranged between 3000 and $3500 \mathrm{~s}^{-1}$. Data was reduced by relating the stresses and strains to the incident, reflected and transmitted strain signals. ${ }^{17}$ Wave dispersion was also corrected, according to the guidelines of Lifshitz and Leber. ${ }^{18}$ Finally, specimen equilibrium was verified by comparing the applied forces on each side of each specimen.

The stress-strain response of dynamically loaded foams with relative densities from 0.13 to 0.60 is presented in Fig. 2. The same general behavior is observed for all relative densities: a peak in stress is attained at approximately $2-3 \%$ strain, followed by relaxation to a fairly constant stress plateau. Expectedly, as the relative density decreases, the modulus, yield stress, and plateau stress all decrease. After failure, the foam appears fairly fragmented. The fragments consist largely of undeformed fractured portions as well as portions that have been plastically deformed to full densification [Fig. 1(b)]. Micrographs in Figs. 1(c) and 1(d) show one such densified fragment. Severely deformed cell walls and regions densely populated with shear bands can be seen in Fig. 1(d), indicating that the struts yielded plastically prior to fracturing. The existence of these plastically deformed features implies that plasticity may be the dominant yielding mechanism during dynamic loading.

The quasistatic loading tests were performed over relative densities between 0.22 and 0.65 . The postyielding behavior of these foams under quasistatic loading has been studied extensively elsewhere. ${ }^{10,11}$ A typical stress strain response of a 0.4 relative density foam deformed under a strain rate of $10^{-4} \mathrm{~s}^{-1}$ is shown in Fig. 3. In the same plot, we also present the response of a specimen of equivalent relative density deformed dynamically under a strain rate of $3500 \mathrm{~s}^{-1}$. The postyielding deformation for the two strain rates present some notable similarities: yielding is followed by a stress drop of about $40 \%$ toward a rough stress plateau that extends beyond $10 \%$ strain. In addition, the yield strength for the two strain rates is nearly identical. This could be expected as monolithic amorphous metals are known to be strain-rate insensitive. ${ }^{13,14}$ For other relative densities however, varying the strain rate results in very different foam yield strengths.

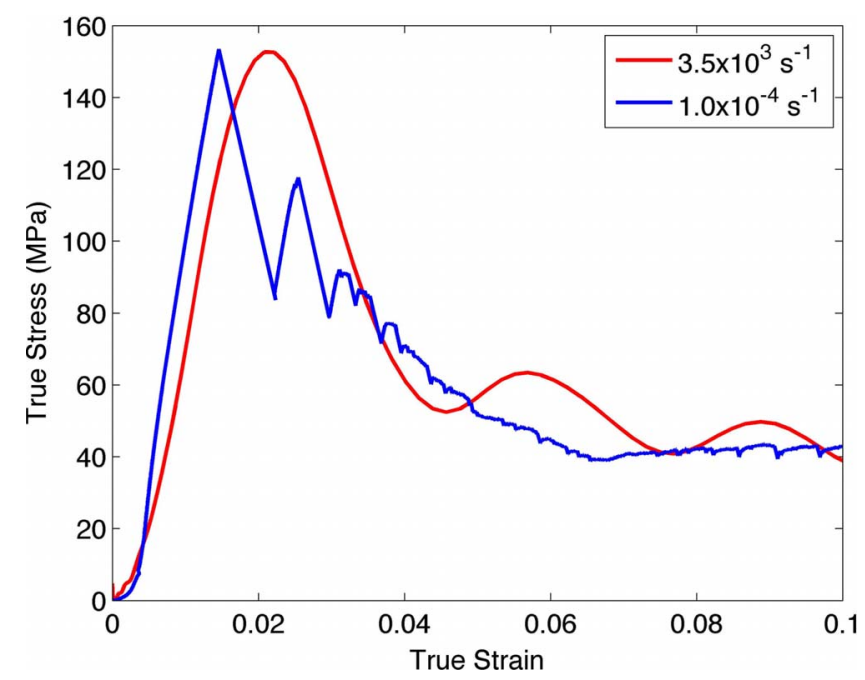

FIG. 3. (Color online) A comparison of the stress-strain response of two $60 \%$ porosity foams under applied strain rates of 3500 and $1 \times 10^{-4} \mathrm{~s}^{-1}$.

In Fig. 4 we plot the relative strength (foam yield strength, $\sigma_{\mathrm{y}}^{*}$, normalized by the solid yield strength, $\sigma_{\mathrm{ys}}$, known to be $1630 \mathrm{MPa})^{11}$ as a function of the relative density, $\rho^{*} / \rho_{\mathrm{s}}$, for the foams tested dynamically along with those tested quasistatically. As seen in the plot, the relative strength versus relative density data for low and high strain rate fall on two distinct curves with different slopes. The two slopes point to two distinct mechanisms of yielding. That is, even though the postyielding behavior for low and high strain rate appears to be qualitatively similar (Fig. 3), the actual yielding transition (i.e., the elastic to plastic transition) appears to be fundamentally different for the two strain rate regimes. Power law fits using $\sigma_{\mathrm{y}}^{*} / \sigma_{\mathrm{ys}} \sim\left(\rho^{*} / \rho_{\mathrm{y}}\right)^{n}$ give $n=2.38$ for the quasistatic data and 1.65 for the dynamic data. In the work of Gibson and Ashby, ${ }^{19}$ strength/porosity relations characterized by a power exponent of $\sim 2$ indicate a foam-yielding mechanism dominated by elastic buckling, while power exponents of $\sim 1.5$ indicate predominantly plastic yielding. The yielding transition of highly stochastic metallic glass foams loaded quasistatically has indeed been identified by $\mathrm{x}$-ray microtomography to be elastic buckling. ${ }^{10}$ Specifically,

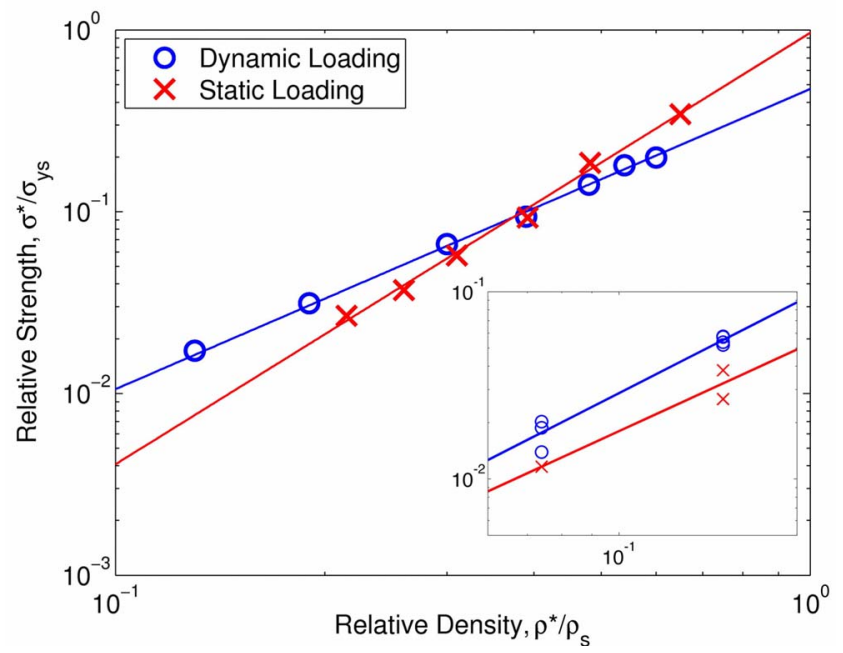

FIG. 4. (Color online) Relative strength plotted against relative density for foams tested under low and high applied strain rates. The inset shows a data for quasistatic $\left(\sim 10^{-4} \mathrm{~s}^{-1}\right)$ and dynamic $\left(10^{3} \mathrm{~s}^{-1}\right)$ compression of aluminum foams (Ref. 19). Solid lines are power-law fits to the data. 
yielding in such foams is found to initiate by Eulerian buckling of high-aspect-ratio membranes distributed randomly throughout the cellular structure, and evolve by percolation of these elastic instabilities toward a noncatastrophic collapse event. Elastic yielding of quasistatically loaded foams is therefore consistent with the relative strength/relative density power exponent of 2.38 . The strength/porosity power exponent of 1.65 for the dynamic test data on the other hand points to a plastic yielding mechanism. The severe plastic deformation observed in the fragments of dynamically loaded foams [Figs. 1(c) and 1(d)] supports that dynamic foam yielding is indeed dominated by plasticity. The strength/porosity correlations, supported by observations of the yielding and failure transitions, suggest that metallic glass foams with essentially self-similar cellular structures yield by different mechanisms when loaded under drastically different strain rates. Conventional metal foams (e.g. aluminum foams), demonstrate higher yield strengths in dynamic loading than in quasistatic loading across the entire relative density spectrum, but show no apparent shift in the strength/ porosity correlation ( Fig. 4 inset). ${ }^{20}$ The higher yield strengths under dynamic loading is a consequence of the strain-rate sensitivity of monolithic aluminum. Fitting the aluminum-foam data to a power law shows that the exponents are very close to the characteristic exponent for plastic yielding of $\sim 1.5$ ( $n=1.4$ for static and 1.6 for dynamic), suggesting that the dominant yielding mechanism for aluminum foams remains unchanged on going from quasistatic to dynamic strain rates.

The strain-rate-induced change in the foam yielding mechanism from elastic buckling to plastic yielding for the metallic glass foams investigated here can be understood by examining the relevant mechanisms and associated time scales. Because of the high elastic limit of metallic glasses, a metallic glass column is generally less stable against buckling for a certain aspect ratio than a crystalline metal column. Specifically, the critical aspect ratio for elastic buckling is given by $k \pi / \sqrt{\varepsilon_{\mathrm{el}}}$, where $\varepsilon_{\mathrm{el}}$ is the elastic strain limit of the material, and index $k$ depends on the end constraints and ranges between $1 / 2$ and $2 .{ }^{21}$ Using $\varepsilon_{\mathrm{el}}=0.02$ for a metallic glass column, one can estimate the critical aspect ratio to range between 20 and 40 . In contrast, the critical aspect ratio for an aluminum column with $\varepsilon_{\mathrm{el}}=0.005$ can be estimated to range between 40 and 80 . This propensity for elastic buckling gives rise to the elastic yielding tendency of these foams. ${ }^{10}$ As known from the work of Lindberg and Florence, ${ }^{22}$ the transient buckling response of a column to dynamic pulsed load is characterized by a time scale associated with the rate of sound-wave propagation across the column. When a column is submitted to a pulsed load for duration shorter than this time scale, or equivalently, when the rate of deformation of a column exceeds this characteristic rate, elastic buckling may be suppressed and the column might yield plastically. To examine this concept as it pertains to this study, we define two time scales: the time scale associated with the rate of sound-wave propagation, $\tau_{\text {wave }}=l / c$, where $l$ is a characteristic length scale and $c$ is the speed of sound in the material, and the time scale associated with the rate of elastic deformation, $\tau_{\text {load }}=\varepsilon_{\mathrm{el}} / \dot{\varepsilon}$, where $\dot{\varepsilon}$ is the ap- plied strain rate. If $\tau_{\text {load }} \gg \tau_{\text {wave }}$, as in quasistatic loading, buckling would be enabled. If $\tau_{\text {load }} \leq \tau_{\text {wave }}$, as in dynamic loading, buckling would be suppressed. For a metallic glass membrane typical of foams in the current study, $l$ is on the order of the average cell size, which is $1 \mathrm{~mm}, c=\sqrt{E_{s} / \rho_{s}}$ $\approx 3200 \mathrm{~m} / \mathrm{s}$, where $E_{s} \approx 100 \mathrm{GPa}$ and $\rho_{s} \approx 10^{4} \mathrm{~kg} / \mathrm{m}^{3}$ are the Young's modulus and density of $\mathrm{Pd}_{43} \mathrm{Ni}_{10} \mathrm{Cu}_{27} \mathrm{P}_{20}$ glass, respectively, and $\varepsilon_{\mathrm{el}} \approx 0.02$. This data give $\tau_{\text {wave }}=3$ $\times 10^{-7}$ s. For a quasistatic loading test with $\dot{\varepsilon}=10^{-4} \mathrm{~s}^{-1}$ we have $\tau_{\text {load }}=200 \mathrm{~s} \gg \tau_{\text {wave }}$, which implies that a stress wave may travel across the membrane many times before the plastic yield strain is reached, therefore elastic buckling would occur. For a dynamic loading test with $\dot{\varepsilon}=10^{4} \mathrm{~s}^{-1}$, we have $\tau_{\text {load }}=2 \times 10^{-6} \mathrm{~s} \cong \tau_{\text {wave }}$, which implies that the plastic yield strain would be reached as soon as the stress wave begins propagating through the membrane, and therefore elastic buckling would be avoided.

In conclusion, microscopic analysis along with strength/ porosity relations for stochastic metallic glass foams loaded dynamically reveal that dynamic yielding is controlled predominantly by plasticity, unlike quasistatic foam yielding, which is known to be controlled by elastic buckling. The strain-rate induced shift in the foam yielding mechanism is attributed to the rate of loading approaching the rate of sound-wave propagation across structural membranes, which suppresses elastic buckling and promotes plastic yielding.

This work was supported in part by the MRSEC Program of the National Science Foundation under Award No. DMR-0520565 and by the Office of Naval Research under Grant No. N00014-07-1-1115.

${ }^{1}$ A. H. Brothers and D. C. Dunand, Scr. Mater. 54, 513 (2006).

${ }^{2}$ A. H. Brothers and D. C. Dunand, MRS Bull. 32, 639 (2007).

${ }^{3}$ M. H. Lee and D. J. Sordelet, Appl. Phys. Lett. 89, 021921 (2006).

${ }^{4}$ J. Schroers, C. Veazey, M. Demetriou, and W. Johnson, J. Appl. Phys. 96, $7723(2004)$

${ }^{5}$ T. Wada, X. Wang, H. Kimura, and A. Inoue, Scr. Mater. 59, 1071 (2008).

${ }^{6}$ M. D. Demetriou, G. Duan, C. Veazey, K. De Blauwe, and W. L. Johnson, Scr. Mater. 57, 9 (2007).

${ }^{7}$ M. F. Ashby and A. L. Greer, Scr. Mater. 54, 321 (2006).

${ }^{8}$ A. H. Brothers and D. C. Dunand, Acta Mater. 53, 4427 (2005).

${ }^{9}$ A. H. Brothers and D. C. Dunand, Adv. Mater. 17, 484 (2005).

${ }^{10}$ M. D. Demetriou, J. C. Hanan, C. Veazey, M. D. Michiel, N. Lenoir, E. Üstündag, and W. L. Johnson, Adv. Mater. 19, 1957 (2007).

${ }^{11}$ T. Wada and A. Inoue, Mater. Trans. 45, 2761 (2004).

${ }^{12}$ M. D. Demetriou, C. Veazey, J. S. Harmon, J. P. Schramm, and W. L. Johnson, Phys. Rev. Lett. 101, 145702 (2008).

${ }^{13}$ H. A. Bruck, A. J. Rosakis, and W. L. Johnson, J. Mater. Res. 11, 503 (1996).

${ }^{14}$ T. Mukai, T. G. Nieh, Y. Kawamura, A. Inoue, and K. Higashi, Scr. Mater. 46, 43 (2002)

${ }^{15}$ M. D. Demetriou, C. Veazey, J. Schroers, J. C. Hanan, and W. L. Johnson, J. Alloys Compd. 434, 92 (2007).

${ }^{16}$ M. D. Demetriou, C. Veazey, J. Schroers, J. C. Hanan, and W. L. Johnson, Mater. Sci. Eng., A 449, 863 (2007).

${ }^{17}$ H. Kolsky, Stress Waves in Solids (Dover, New York, 1963).

${ }^{18}$ J. Lifshitz and H. Leber, Int. J. Impact Eng. 15, 723 (1994).

${ }^{19}$ L. J. Gibson and M. F. Ashby, Cellular Solids: Structure \& Properties, 2nd ed. (Cambridge University Press, Cambridge, UK, 1997), Chap. 5.

${ }^{20}$ K. A. Dannemann and J. Lankford, Mater. Sci. Eng., A 293, 157 (2000).

${ }^{21}$ J. M. Gere and S. P. Timoshenko, Mechanics of Materials, 3rd ed. (PWSKent, Boston, 1975), Chap. 9.

${ }^{22}$ H. E. Lindberg and A. L. Florence, Dynamic Pulse Buckling: Theory and Experiment (M. Nijhoff, Dordrecht, Boston, 1987) 